\title{
tbg - a new file format for genomic data
}

\section{Philipp Schönnenbeck ${ }^{1}$, Tilman Schell ${ }^{2}$, Susanne Gerber ${ }^{1,{ }^{\star}}$ and Markus Pfenninger $2,3,4^{*}$}

${ }^{1}$ Institute of Human Genetics, University Medical Center, Johannes Gutenberg University, Mainz, Germany

2 Department of Molecular Ecology, Senckenberg Biodiversity and Climate Research Centre, Frankfurt am Main, Germany

${ }^{3}$ Institute for Molecular and Organismic Evolution, Johannes Gutenberg-University Mainz, Johann-Joachim-Becher-Weg 7, 55128,

Mainz, Germany

${ }^{4}$ LOEWE Centre for Translational Biodiversity Genomics, Senckenberg Biodiversity, and Climate Research Centre,

Senckenberganlage 25, 60325, Frankfurt am Main, Germany

*To whom correspondence should be addressed.

Associate Editor: XXXXXXX

Received on XXXXX; revised on XXXXX; accepted on XXXXX

\begin{abstract}
Motivation: The question of determining whether a Single-Nucleotide Polymorphism (SNP) or a variant in general leads to a change in the amino acid sequence of a protein coding gene is often a laborious and time-consuming challenge. Here, we introduce the tbg file format for storing genomic data and tbg-tools, a user-friendly toolbox for the faster analysis of SNPs. The file format stores information for each nucleotide in each gene, allowing to predict which change in the amino acid sequence will be caused by a variant in the nucleotide sequence. Our new tool therefore has the potential to make biological sense of the unprecedented amount of genome-wide genetic variation that researchers currently face.

Results: The new tab-separated file for storing the nucleotide data can be easily analyzed and used for a wide variety of biological research. It is also possible to automate some of these analyses using the additional analysis tools from tbg-tools
\end{abstract}

Availability: tbg-tools is written in Python and allows the installation from the command line. It can be found on https://github.com/Croxa/tbg-tools.

Contact: pschoenn@students.uni-mainz.de

\section{Introduction}

As sequencing methods become faster and cheaper (Kris A. Wetterstrand (2020)), there are ever-increasing amounts of genomic data and new processing possibilities. The analysis of genomic variants, for instance, marks an essential step in many different areas of biomedicine and medical diagnostics and enhances substantial progress in biological research. To determine a species' genomic diversity, variant calling with different individuals' sequencing data is utilized. Either reads of single individuals (individual reseq) or reads of whole populations (poolseq) within a species are sequenced and then compared to the reference genome. These variants are often stored in vcf files (Variant Call Format). It is, for example, possible to associate phenotypes to specific SNPs, for studying the effects of climate change on genomes (Pauls et al (2012), Pfenninger et al (2020)), or to study mutations and changes in the number of genome copies in cancer cells (Koboldt et al., 2012). In this fast growing world of genomics, more and more important and useful new programs and file formats are therefore developed, such as (Li et al (2020)) or GIANT (Vandel et al (2020)). However, many of these programs serve a particular niche and furthermore, they lack of answering more general questions about the sequenced data, such as e.g. providing information about synonymous and non-synonymous substitutions. However, this information is critical for diagnostics and biological research, since non-synonymous mutations can often explain phenotype variants.

To close this gap in currently existing tools, we developed the user-friendly toolbox tbg-tools available on Github as open source. The program offers a built-in toolbox as well as a new data format called $t b g$.tsv. Using the new format, information on specific variants can be accessed quickly and easily using unix command tools like awk (Aho et al (1987)) or with tbg-tools itself. tbg-tools offers a simple command-line based handling and can be 
scaffold position base in reference coding base triplet position amino acid coded gene-ID 4ds to-A to- $\mathrm{T}$ to-C to-G

\begin{tabular}{|c|c|c|c|c|c|c|c|c|c|c|c|}
\hline scaffold001 & 190 & A & A & 1 & M & gene 001 & False & M & $\mathrm{L}$ & $\mathrm{L}$ & V \\
\hline \multicolumn{12}{|c|}{$\begin{array}{l}\text { scaffold } 001 \text { 191 } \\
\text { bioRxiv pre } \\
\text { scaffold00this prep }\end{array}$} \\
\hline scaffold001 & 193 & A & $\mathrm{A}$ & 1 & permission & gene001 & False & K & stop & Q & $\mathrm{E}$ \\
\hline scaffold001 & 194 & $\mathrm{~A}$ & $\mathrm{~A}$ & 2 & K & gene001 & False & $\mathrm{K}$ & I & $\mathrm{T}$ & $\mathrm{R}$ \\
\hline scaffold001 & 195 & A & $\mathrm{A}$ & 3 & K & gene001 & False & $\mathrm{K}$ & $\mathrm{N}$ & $\mathrm{N}$ & K \\
\hline scaffold001 & 196 & $\mathrm{C}$ & $\mathrm{C}$ & 1 & $\mathrm{R}$ & gene001 & False & $\mathrm{S}$ & $\mathrm{C}$ & $\mathrm{R}$ & G \\
\hline scaffold001 & 197 & G & G & 2 & $\mathrm{R}$ & gene001 & False & $\mathrm{H}$ & $\mathrm{L}$ & $\mathrm{P}$ & $\mathrm{R}$ \\
\hline
\end{tabular}

Table 1. Example for a tbg.tsv file. For each nucleotide of each gene there is one row with twelve columns. These columns carry the following information: Scaffold refers to the ID of the sequence on which the current gene is located. Position gives the position of the nucleotide on the sequence defined by the scaffold. The position is 1-based, like the gff files. base in reference provides the base located at the coordinate in the sequence. Coding base is located in the gene. If the gene is on the same strand as the reference sequence, this column is equal to the base in reference column. Otherwise, it is the complementary base. Triplet position is the position in the current encoding triplet. Possible positions are therefore 1,2 or 3 , respectively. Amino acid coded is the amino acid encoded by the current triplet. Gene ID provides the gene-ID of the current gene. If no substitution of the current bases leads to a change of the amino acid, the column $\mathbf{4 d}$ is "True" - otherwise, it is "False". The last four columns, to-A, to-T, to-C and to-G give the amino acids expected when replacing the current base with A, T, C or G.

installed within seconds using GitHub. It requires a Python version of 3.6 or higher but no additional modules that might complicate the installation. For analysis tbg-tools only need a fasta file with the genome and an associated gff file with the genome's coding regions. Since these data files are usually created during de-novo genome assembly and -annotation the creation of a $t b g$ file can be easily integrated into an already existing analysis pipeline.

\section{Methods}

The new file format tbg.tsv is a tab-separated table that can be written as output by tbg-tools (Table 1). This human readable $t s v$ file is designed for manual editing and analysis by the user. Internally, tbg-tools works with the binary $t b g$ format, which is designed and optimized with respect to storage space. tbg-tools can use this optimized file format for extracting specific positions in $c s v, v c f$ and $s y n c$ files. The output is a modified version of these files with information about the coded amino acid.

As an example, for the run-time (figure S1) and the maximum RAM usage (figure S2), tbg-tools was challenged with the data from the human genome project version GRCh38 using a different amount of threads each time. The given fasta file has a size of $3.1 \mathrm{~GB}$, the gff file has $1.1 \mathrm{~GB}$ and the $v c f$ file used has a size of $0.27 \mathrm{~GB}$. tbg-tools also has an option for computers with a lower amount of RAM.

While analyzing $v c f$ files, a stats file option exists, which provides the user with some additional statistics about their $v c f$ file (see Table S1). These statistics include, among other things the number of positions found in coding regions, the number of synonymous and non-synonymous changes on all chromosomes and the number of variants that are not SNPs (Indels). The additional stats files for each individual of a $v c f$ file have some further information for each individual.

\section{Discussion}

We expect a broad field of applications to benefit from using the $t b g$.tsv file with minimal manual effort. An example for the analysis of synonymous and non-synonymous mutations using tbg-tools is the calculation and interpretation of the $\omega=K_{a} / K_{s}$ ratio (also known as the $\mathrm{dN} / \mathrm{dS}$ ratio) (Motoo Kimura (1977)). Another potential field of application could be the search for specific amino acid changes during medical diagnostics. If any amino acid of the $\alpha$-helix is substituted by a proline, the helix is most often interrupted, and the functionality of the protein is not guaranteed anymore. Therefore, it could become routine part of the diagnostics analyses to search for those specific substitutions in the amino acid sequence that could cause structural changes or a stop codon.

Because the tbg.tsv file has a column indicating 4-degenerate sites it can be utilized to identify these positions. 4-degenerate sites can be used e.g. in the calculation of the site frequency spectrum at neutral sites (Chueca et al (2021)) and guide the detection of positive selection in coding sequences (Künstner et al (2010)). Overall, tbg-tools and the new format tbg.tsv are versatile for various analyses and can be easily added to existing genome analysis pipelines without consuming much time while still being easy to install and use.

Acknowledgement The authors acknowledge funding from the Emergent AICenter funded by the Carl-Zeiss-Stiftung and thank the LOEWE-Centre TBG funded by the Hessen State Ministry of Higher Education, Research and the Arts (HMWK).

\section{References}

Baxel Künstner and Benoit Nabholz and Hans Ellegren (2010), Significant Selective Constraint at 4-Fold Degenerate Sites in the Avian Genome and Its Consequence for Detection of Positive Selection, 10.1093/gbe/evr112, Genome Biology and Evolution, 3, 1381-1389.

Motoo Kimura, Preponderance of synonymous changes as evidence for the neutral theory of molecular evolution, 10.1038/267275a0,Nature, 267, 275-276.

Aho, Alfred V. and Kernighan, Brian W. and Weinberger, Peter J., The AWK Programming Language, ISBN: 020107981X, Addison-Wesley Longman Publishing Co., Inc., USA

Jimmy Vandel and Gheeraert, Céline and Jérôme Eeckhoute and Bart Staels and Philippe Lefebvre and Julie Dubois-Chevalier, GIANT: galaxy-based tool for interactive analysis of transcriptomic data, 10.1038/s41598-020-76769-w, Scientific Reports, 10, 275-276.

Steffen U. Pauls and Carsten Nowak and Miklós Bálint and Markus Pfenninger, The impact of global climate change on genetic diversity within populations and species, 10.1111/mec.12152, Molecular Ecology, 22(4), 925-946

Jun $\mathrm{Li}$ and $\mathrm{Hu}$ Chen and Yumeng Wang and Mei-Ju May Chen and Han Liang, Next-Generation Analytics for Omics Data, 10.1016/j.ccell.2020.09.002, Cancer Cell.

Kris A. Wetterstrand,DNA Sequencing Costs: Data from the NHGRI Genome Sequencing Program (GSP), https://www.genome.gov/about-genomics/factsheets/DNA-Sequencing-Costs-Data

Markus Pfenninger and Friederike Reuss and Angelika Kiebler and Philipp Schönnenbeck and Cosima Caliendo and Susanne Gerber and Berardino Cocchiararo and Sabrina Reuter and Nico Blüthgen and Karsten Mody and Bagdevi Mishra and Miklós Bálint and Marco Thines and Barbara Feldmeyer, Genomic basis of drought resistance in Fagus sylvatica, 10.1101/2020.12.04.411264

Chueca LJ, Schell T and Pfenninger M, Speciation and historical demography of the land snail sister species Candidula unifasciata and C. rugosiuscula 